\title{
Use of apparent diffusion coefficients in evaluating the response of vestibular schwannomas to Gamma Knife surgery
}

\author{
Clinical article
}

\author{
Chun-Chao Chuang, M.S., ${ }^{1,4,5}$ Cheng-Siu Chang, M.D., ${ }^{2,3}$ Yu-Sheng Tyan, M.D., ${ }^{1,4}$ \\ Keh-Shit Chuang, Ph.D., ${ }^{5}$ Hsien-Tang Tu, B.S., ${ }^{6}$ and Chuan-Fu Huang, M.D. ${ }^{6,7}$ \\ ${ }^{1}$ School of Medical Imaging and Radiological Sciences, ${ }^{2}$ School of Medicine, ${ }^{3}$ Department of \\ Neurosurgery, and ${ }^{4}$ Department of Radiology, Chung Shan Medical University, Taichung; ${ }^{5}$ Department of \\ Biomedical Engineering and Environmental Sciences, National Tsing Hua University, Hsinchu; ${ }^{6}$ School of \\ Medicine, China Medical University; and ${ }^{7}$ Department of Neurosurgery, China Medical University \\ Hospital, Taichung, Taiwan
}

Object. Cellular density is a major factor responsible for changes in apparent diffusion coefficients (ADCs). The authors hypothesized that loss of tumor cells after Gamma Knife surgery (GKS) might alter ADC values. Magnetic resonance imaging, including diffusion-weighted (DW) imaging, was performed to detect cellular changes in brain tumors so that the authors could evaluate the tumor response to GKS as well as the efficacy of the procedure.

Methods. The authors conducted a prospective trial involving 31 patients harboring solid or cystic vestibular schwannomas (VSs) that were treated with GKS. The patients underwent serial MR imaging, including DW imaging, before GKS and at multiple intervals following the procedure. The authors observed the patients over time, evaluating MR imaging findings and clinical outcomes at 6-month intervals. The ADCs were calculated from echo-planar DW images, and mean ADC values were compared at each follow-up.

Results. The mean follow-up period was 36.5 months (range 18-60 months). Imaging studies showed a reduction in tumor volume in 19 patients $(61.3 \%)$ and tumor growth arrest in 9 patients (29\%). In the remaining 3 patients $(9.7 \%)$, tumor enlargement was documented at 18,36, and 42 months.

The mean ADC value before GKS for all solid VSs was $1.06 \pm 0.17 \times 10^{-3} \mathrm{~mm}^{2} / \mathrm{second}$, which significantly increased 6 months after GKS and continued to increase with time $(\mathrm{p}=0.0086)$. The mean ADC value for treated solid tumors as of the last mean follow-up of 36 months (range 18-60 months) was $1.72 \pm 0.26 \times 10^{-3} \mathrm{~mm}^{2} / \mathrm{second}$ (range $\left.1.50-2.09 \times 10^{-3} \mathrm{~mm}^{2} / \mathrm{second}\right)$, which was significantly higher than that before GKS $(\mathrm{p}=0.0001)$. Tumor volumes were positively related to ADC values $(\mathrm{p}=0.03)$.

The mean ADC value before GKS for all cystic VSs was $2.09 \pm 0.24 \times 10^{-3} \mathrm{~mm}^{2} / \mathrm{second}$ (range 1.80-2.58 $\times 10^{-3}$ $\mathrm{mm}^{2} / \mathrm{second}$ ). The mean ADC value for treated cystic tumors as of the last mean follow-up of 38 months (range 18-48 months) was $1.89 \pm 0.22 \times 10^{-3} \mathrm{~mm}^{2} /$ second.

In 3 patients harboring solid VSs, the tumor enlarged after GKS but the ADC values were higher than those before GKS. The authors considered these tumors to be controlled and continued follow-up in the patients.

Conclusions. Apparent diffusion coefficient values may be useful for evaluating treatment results before any definite volume change is detected on imaging studies and for distinguishing radiation-induced necrosis from tumor recurrence in cases in which other imaging results are not definitive, as in cases of increased tumor volume or no volume change. The authors suggest that ADC measurements be included during routine MR imaging examinations for the evaluation of GKS results.

(http://thejns.org/doi/abs/10.3171/2012.7.GKS121003)

\section{KEY Words - vestibular schwannoma - stereotactic radiosurgery • magnetic resonance imaging • diffusion-weighted imaging • apparent diffusion coefficient}

$\mathrm{R}$ ADIOSURGERY, a precise and accurate procedure in which radiation is delivered to the defined target in one step, often produces a powerful radiological effect. ${ }^{10}$ Radiosurgery is performed in patients with

Abbreviations used in this paper: $\mathrm{ADC}=$ apparent diffusion coefficient; DW = diffusion-weighted; GKS = Gamma Knife surgery; $\mathrm{VS}=$ vestibular schwannoma.

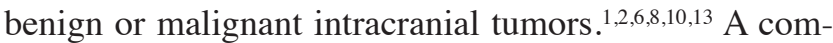
mon approach used to estimate tumor size is to measure the 3 major axes of the tumor on MR images. A positive treatment response is typically defined as a substantial decrease in tumor size or no increase in the cross-sectional area of the tumor. Although radiosurgery substantially decreases tumor mass, the process is time consuming because shrinkage may proceed relatively slowly with 
absorption of cellular debris. ${ }^{3}$ An examination of the literature shows that the tumor control rate for VSs treated with GKS is greater than $90 \%$, but a significant proportion of these tumors $(19.6 \%-51 \%)$ display no change in size.$^{6,8,13}$ Even though no change in tumor size throughout long-term follow-up is also considered successful tumor control, it is likely that the cellularity of the tumor has already been altered after treatment.

Cellularity and the integrity of cellular membranes that impede water translational mobility can affect the diffusion of water within tumor tissues. Diffusionweighted imaging with echo-planar sequences can be used to differentiate various brain tumors, ${ }^{9,12,14,16}$ but it is rarely used to evaluate tumor responses after treatment. ${ }^{4,7}$ There have been no reports on changes in VSs after GKS. The purpose of this study was to investigate the clinical usefulness of ADC values for evaluating VSs and to assess the correlation of DW imaging measurements with radiosurgical effects on the tumors.

\section{Methods}

\section{Patient Population}

From January 2004 to December 2008, we conducted a prospective trial in 31 patients who harbored solid (24 patients) or cystic (7 patients) VSs and were treated by GKS performed with a 201-cobalt source at our Gamma Knife Center. The study was approved by the local ethics committee, and informed consent was obtained from the patients. Routine MR imaging sessions, in which DW images and ADC measurements for the VSs were obtained, were regularly scheduled before GKS and at 6-month intervals following GKS.

The clinical data obtained in the 31 patients are shown in Table 1.

Following administration of a local anesthetic agent supplemented with a sedative, each patient's head was fitted with a Leksell model G stereotactic coordinate frame (Elekta Instruments). High-resolution contrast-enhanced MR imaging was performed to determine tumor location. The GammaPlan dose-planning system (Elekta) was used to determine dosimetry with multiple isocenters. In this study the mean tumor volume was $8.2 \mathrm{~cm}^{3}$ (range $1-24 \mathrm{~cm}^{3}$ ), and the mean tumor margin dose was $12 \mathrm{~Gy}$ (range 11-13 Gy).

\section{Apparent Diffusion Coefficients}

Magnetic resonance images were obtained in all patients by using a 1.5-T scanner (Signa Horizon LX2, GE Medical Systems). The manufacturer-supplied birdcage 8-channel neurovascular coil was used to detect signals during conventional MR and DW imaging. The DW imaging parameters were as follows: TE $95 \mathrm{msec}$; TR 10,000 msec; matrix size $128 \times 128$; FOV $300 \mathrm{~mm}$; slice thickness $5.5 \mathrm{~mm}$; NEX 1. Diffusion was measured in 3 directions (along the $\mathrm{x}, \mathrm{y}$, and $\mathrm{z}$ axes), with $\mathrm{a} \mathrm{b}$ value of 1000 seconds $/ \mathrm{mm}^{2}$ for each direction. In addition, an image without diffusion gradients was acquired. The total acquisition time for all images with various diffusion sensitivities was 40 seconds. To minimize the effect of diffusion anisotropy, an average of the values obtained
TABLE 1: Clinical characteristics in 31 patients with VSs

\begin{tabular}{|c|c|c|}
\hline Characteristic & Solid VSs (24 patients) & $\begin{array}{l}\text { Cystic VSs } \\
\text { (7 patients) }\end{array}$ \\
\hline patient age $(y r s)^{*}$ & $51(18-66)$ & $59(44-77)$ \\
\hline female/male ratio & $14: 10$ & $4: 3$ \\
\hline tumor volume $\left(\mathrm{cm}^{3}\right)^{*}$ & $8.2(1-24)$ & $9.9(3.4-16.1)$ \\
\hline margin dose $(G y)^{*}$ & $12(11-13)$ & $11.6(11-12)$ \\
\hline follow-up (mos) ${ }^{*}$ & $36(18-60)$ & $38(18-48)$ \\
\hline \multicolumn{3}{|l|}{ tumor size† } \\
\hline decreased & $13(54.2)$ & $6(85.7)$ \\
\hline no change & $8(33.3)$ & $1(14.3)$ \\
\hline increased & $3(12.5)$ & $0(0)$ \\
\hline \multicolumn{3}{|c|}{ ADC value $\left(\times 10^{-3} \mathrm{~mm}^{2} / \mathrm{sec}\right)$ showing successful tumor control } \\
\hline pre-GKS & $1.06 \pm 0.17$ & $2.09 \pm 0.24$ \\
\hline post-GKS & $1.72 \pm 0.26$ & $1.89 \pm 0.22$ \\
\hline increased size & $1.52 \pm 0.16$ (3 patients) & \\
\hline
\end{tabular}

for the 3 diffusion directions was calculated to provide the trace of the diffusion tensor.

\section{Analysis of the MR Imaging Data}

Image postprocessing was performed using a workstation running vendor-provided software (Advantage 4.0 and FuncTool, respectively; GE Medical Systems). These software programs enable visualization and processing of DW imaging data. We created maps of the ADC values by performing calculations on a pixel-by-pixel basis. Data were analyzed using a t-test to compare the mean ADC values obtained before GKS with those obtained after GKS. A p value $<0.05$ was considered statistically significant.

\section{Results}

\section{Magnetic Resonance Imaging}

The mean follow-up period in this study was 36.5 months (range 18-60 months). Imaging studies demonstrated a reduction in tumor volume in 19 patients $(61.3 \%)$ and tumor growth arrest in 9 patients (29\%) (Table 1). There was tumor enlargement at 18,36 , and 42 months in the remaining 3 patients (9.7\%). Imaging studies revealed a loss of central enhancement of the tumor in 28 patients (90.3\%). The volume of central necrosis paralleled the reduction in tumor size.

\section{Apparent Diffusion Coefficients}

The mean ADC value for all solid VSs was $1.06 \pm$ $0.17 \times 10^{-3} \mathrm{~mm}^{2} / \mathrm{second}$ (range $0.97-1.27 \times 10^{-3} \mathrm{~mm}^{2} / \mathrm{sec}-$ ond). This value significantly increased 6 months after GKS to $1.57 \pm 0.2 \times 10^{-3} \mathrm{~mm}^{2} / \mathrm{second}$ and continued to increase with time $(\mathrm{p}=0.0086)$. The mean $\mathrm{ADC}$ value for treated solid VSs as of the last follow-up (mean 36 


\section{Evaluating the response and efficacy of VSs after GKS}

months, range $18-60$ months) was $1.72 \pm 0.26 \times 10^{-3} \mathrm{~mm}^{2} /$ second, which was significantly higher than the value obtained before GKS ( $\mathrm{p}=0.0001)$ (Fig. 1). Tumor volumes were positively related to ADC values $(p=0.03)$. Changes in ADC values for the 24 solid VSs ranged from $26 \%$ to $90 \%$ (mean $48 \% \pm 17 \%$ ), and changes in ADC values for the 7 cystic VSs ranged from $-32 \%$ to $17 \%$ (mean $-13 \%$ $\pm 16.7 \%$ ) (Fig. 2). In 3 patients who harbored solid VSs, the tumor enlarged after GKS, but the ADC values were higher than those measured before GKS. We considered these tumors to be controlled and continued follow-up in these 3 patients (Fig. 3).

The mean ADC value for all cystic VSs before GKS was $2.09 \pm 0.24 \times 10^{-3} \mathrm{~mm}^{2} / \mathrm{second}$ (range 1.80-2.58 $\times$ $10^{-3} \mathrm{~mm}^{2} / \mathrm{second}$ ). The mean ADC value for treated cystic VSs as of the last mean follow-up of 38 months (range $18-48$ months) was $1.89 \pm 0.22 \times 10^{-3} \mathrm{~mm}^{2} / \mathrm{second}$, which showed a tendency to decrease compared with that before GKS ( $p=0.129$ ) (Fig. 4). The pre-GKS ADC values for cystic tumors were significantly higher than those for solid tumors ( $\mathrm{p}<0.001)$, and the downward trend in cystic tumor values also differed from the upward trend in solid tumor values after GKS. Changes in ADC values ranged from $+17 \%$ to $-32 \%$ (mean $-8.4 \%$ ) (Fig. 2).

With regard to changes in tumor volumes, the mean ADC values were as follows: tumor-volume reduction group, $1.75 \pm 0.19 \times 10^{-3} \mathrm{~mm}^{2} / \mathrm{second}$ (range 1.36-2.08 $\times 10^{-3} \mathrm{~mm}^{2} / \mathrm{second}$ ); no tumor-volume change group, 1.77 $\pm 0.20 \times 10^{-3} \mathrm{~mm}^{2} / \mathrm{second}$ (range $1.39-2.08 \times 10^{-3} \mathrm{~mm}^{2} /$ second); and tumor-volume increase group, $1.52 \pm 0.16$ $\times 10^{-3} \mathrm{~mm}^{2} /$ second (range $1.47-1.63 \times 10^{-3} \mathrm{~mm}^{2} /$ second) .
No significant differences in ADC values were noted between groups $(\mathrm{p}=0.24)$.

\section{Discussion}

\section{Changes in ADCs for VSs After GKS}

We found that ADCs for solid VSs were relatively higher than ADCs for normal parenchyma or meningiomas-a finding that has also been noted in other studies. ${ }^{14,15,17}$ The high ADC values for the VSs were consistent with relatively low nucleus-to-cytoplasm ratios and increased diffusion rates, indicating the presence of increased amounts of extracellular water (relatively loose tissue) within the tumor matrix. ${ }^{14}$ Histological characteristics may explain these findings, because schwannomas comprise Antoni Type A and Type B neurinomas, and their higher ADCs may reflect the lower cell density of Antoni Type B neurinomas. A subgroup of our patient with cystic schwannomas had significantly high ADC levels, which reflect the tumor's fluid component with a high velocity of water molecules.

After GKS, the ADC values for solid VSs significantly increased from the first follow-up at 6 months and continued to display an upward trend at later followups. Routine MR images showed that most tumors had a loss of central enhancement, but their sizes remained the same or even increased temporarily at some point. Examination of signal intensity on DW images and measurements of ADCs may be useful for monitoring responses after treatment, particularly prior to any change
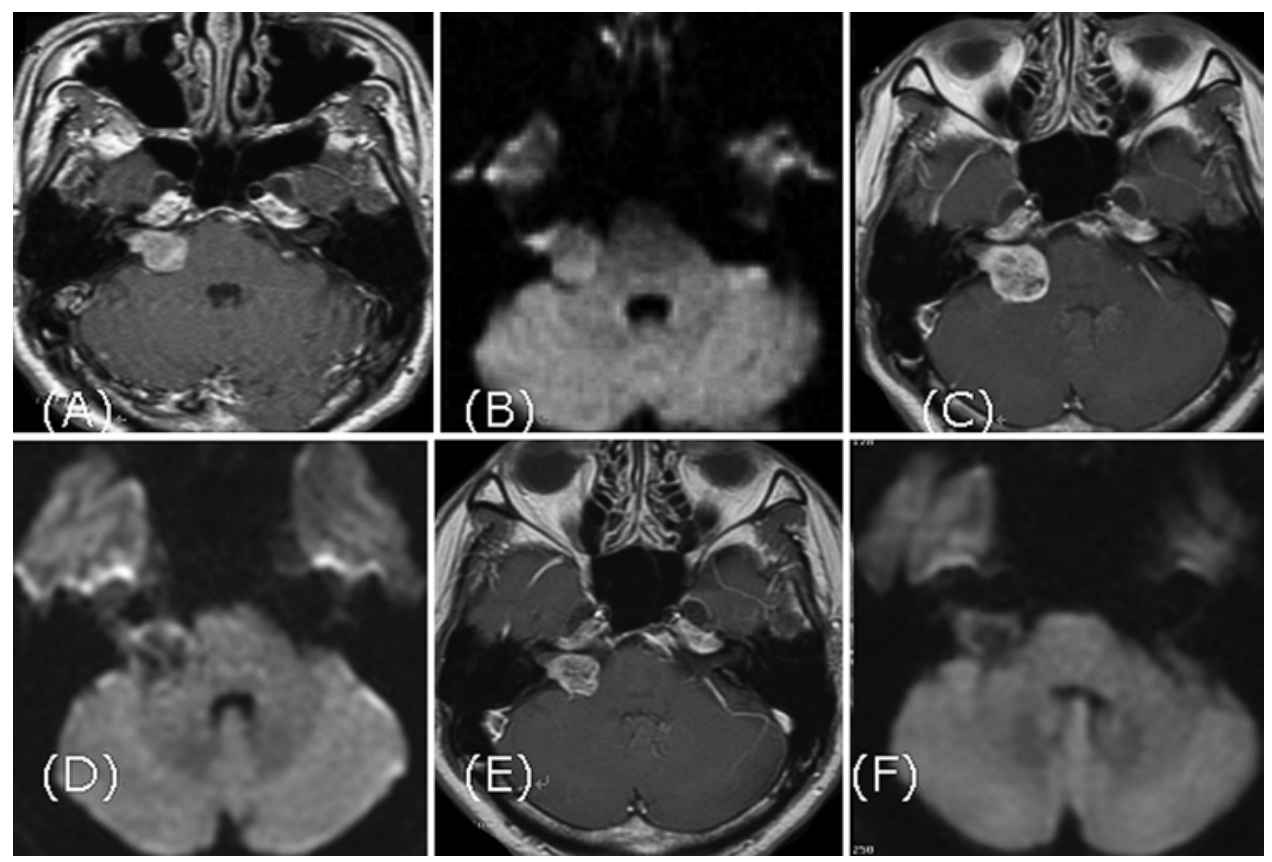

FIG. 1. Magnetic resonance images. A: A Gd-enhanced T1-weighted image showing a solid VS. B: A DW image obtained before GKS displaying mild hypointensity in the region of the tumor ( $A D C=1.07 \pm 0.12 \times 10^{-3} \mathrm{~mm}^{2} / \mathrm{second}$ ). $\quad$ C: At the 12-month follow-up, the tumor volume temporarily increased, but the MR image displays relative hypointensity on the center of the tumor. D: A DW image; the ADC value has increased to $1.65 \pm 0.23 \times 10^{-3} \mathrm{~mm}^{2} / \mathrm{second}$. E: Image showing the shrunken tumor at the 2-year follow-up. F: A DW image displaying hypointensity; the ADC value is $1.40 \pm 0.23 \times 10^{-3} \mathrm{~mm}^{2} / \mathrm{second}$. 


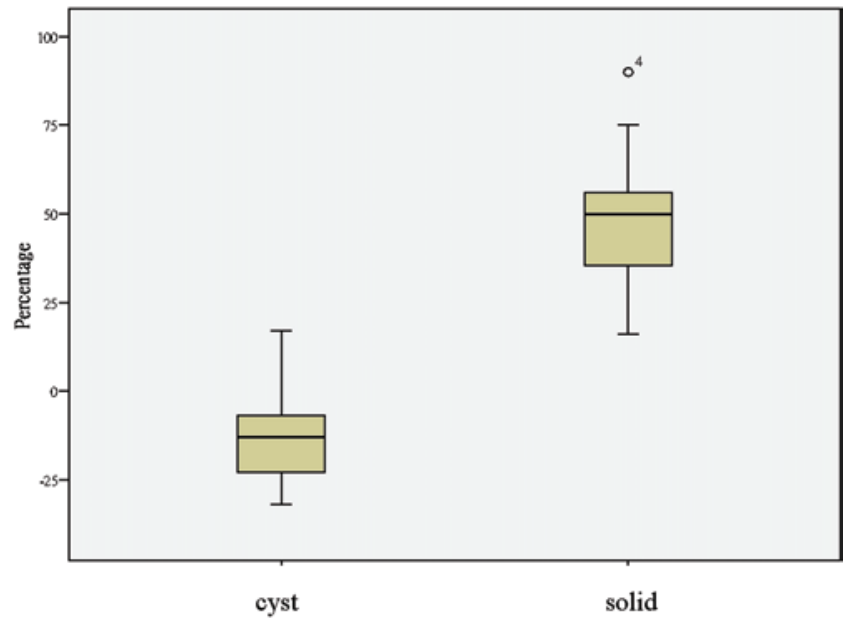

FIG. 2. Bar-and-whisker graph showing the percentage of changes in ADCs before and after GKS in solid (24 patients) and cystic (7 patients) VSs. $\mathrm{O}^{4}$ indicates 4 outliers.

in tumor volume. Other treatments such as chemotherapy, radiotherapy, antigene therapy, or a combination of these treatment modalities may significantly alter the tissue structure of a tumor; therefore, use of ADCs to monitor the cytotoxic response of a tumor to treatment has been recognized in some studies. ${ }^{3,7}$ Use of these therapies consistently leads to increased water diffusion within the tumor, demonstrating a favorable response to treatment. Concordantly, our studies on patients with VSs treated with GKS showed similar diffusion behavior, suggesting a useful application of MR imaging in patients with VSs following GKS. The radiological effect on schwannomas and other benign neoplasms is a combination of both cytotoxic and delayed vascular effects. Linskey et al. ${ }^{11}$ grafted human VSs into the subrenal capsule of nude mice and stated that a reduction in tumor size was due to neoplastic cell death after radiosurgery. We believe the effects of GKS are partly caused by radiation-induced apoptosis or necrosis, especially in treatments in which a low radiation dose is used.

The ADC values for cystic VSs were higher than those for solid tumors, because in the former there was free movement of water molecules. Changes in ADC values for cystic tumors after GKS were opposite those for solid tumors-in all but 1 case there was a trend toward decreased values due to the diminishing cystic component as the tumor shrank.

\section{Clinical Application and Limitation of Changes in ADCs}

In this study, ADC values of solid tumors significantly increased, even before a change in tumor volume was evident. In a previous study ${ }^{5}$ we demonstrated that ADC changes occurred in cases of metastatic tumors as early as 7 days and in cases of meningioma at 30 days after GKS. The rate of change in ADC values in cases of VS needs further intensive study. Nevertheless, we can say that ADC changes precede the real change in tumor size detected on routine follow-up MR images.

In 1 patient with a cystic tumor, the solid portion of the tumor enlarged while the ADC values remained high. Although a recurrence was suspected in this case, we considered the findings to be an effect of radiation; later follow-up proved that treatment had been successful and resulted in remarkable tumor shrinkage (Fig. 4).

At the last follow-up, images in 3 patients showed tumor enlargement. Although we classified these cases as enlarged tumors, the ADC values remained high, and hence, we considered the findings to be radiation-induced effects rather than tumor recurrence. The follow-up period in these 3 patients ranged from 18 to 48 months. For patients with late or temporarily swelling tumors, ADCs may be good indicators to differentiate tumor recurrence from enlargement due to a radiation effect.

Diffusion-weighted imaging of the entire brain can be completed in 3 seconds or in 48 seconds when 7 different diffusion sensitivities (maximum $b=1000$ seconds/ $\mathrm{mm}^{2}$ ) are used. Our study demonstrates the feasibility of performing serial DW imaging for the clinical management of patients with brain tumors.

Values of ADCs can be affected by metal; the lack of magnetic properties of titanium makes it a better choice for the fixation of bone flaps or dental prostheses. The presence of osseous structures near tumors can also cause blurred MR images, but the use of more contemporary high-field Tesla technology may overcome this defect. Because free water mobility increases greatly and extensively relative to tumor cells, more regions of interest can occur, and consequently, the standard deviations of ADCs can be affected. A new generation of software, however,
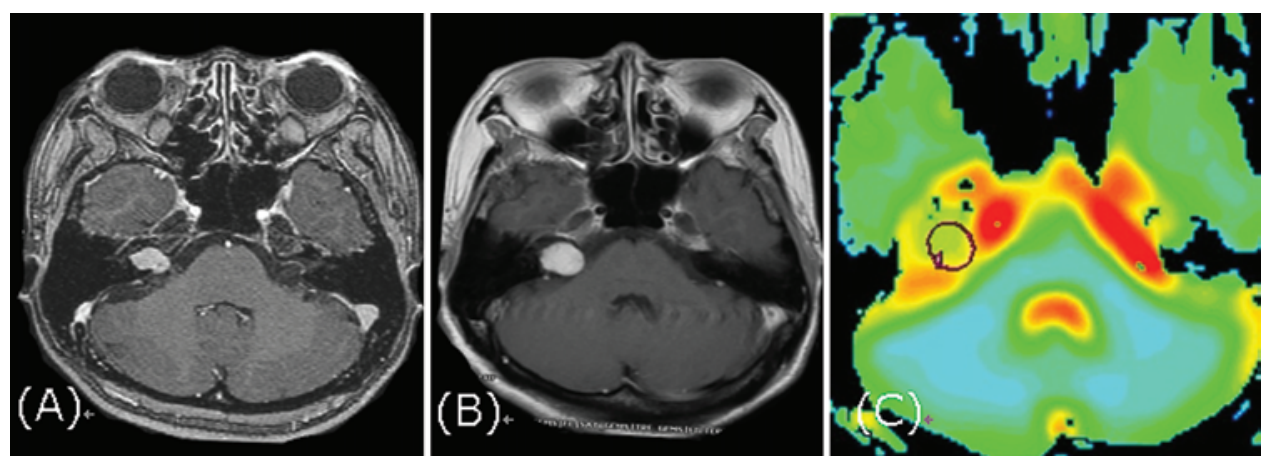

FIG. 3. A: An MR image depicting a VS before GKS. B: An MR image showing the enlarged tumor at the 4-year followup. $\mathrm{C}$ : An $\mathrm{ADC}$ map ( $\left.\mathrm{ADC}=1.50 \pm 0.13 \times 10^{-3} \mathrm{~mm}^{2} / \mathrm{second}\right)$. The circle marks the tumor's location. 

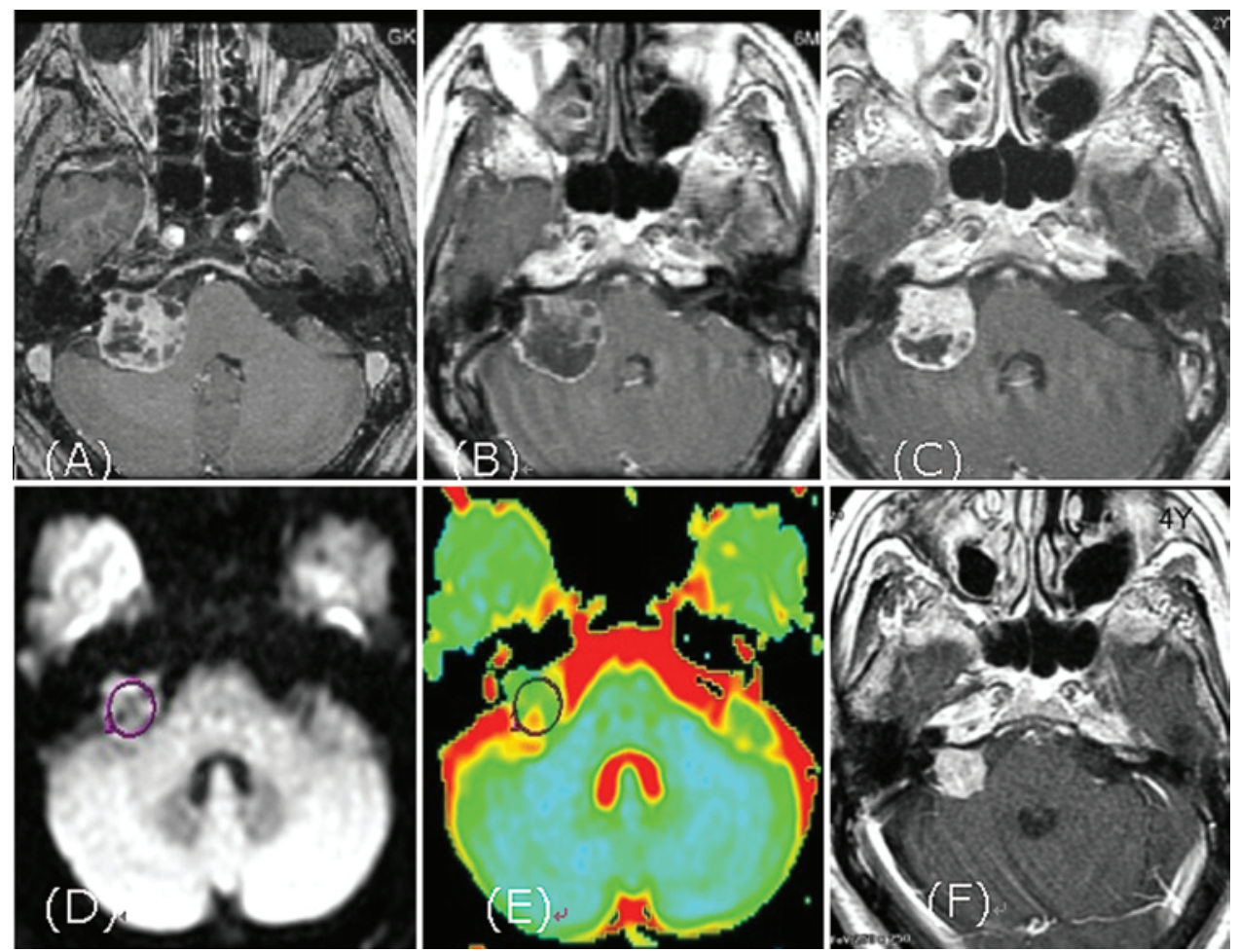

FIG. 4. A-D: Magnetic resonance images. A: A cystic VS is seen on the right side $\left(A D C=1.98 \pm 0.20 \times 10^{-3} \mathrm{~mm}^{2} / \mathrm{sec}^{-}\right.$ ond). B: Marked loss in tumor enhancement is apparent after 1 year ( $A D C=2.16 \pm 0.23 \times 10^{-3} \mathrm{~mm}^{2} /$ second). C: An increase is seen in the enhanced solid portion of tumor at the 2-year follow-up. D: A DW image showing hypointensity (circle) and the region of interest for $A D C$ measurement. $E$ : $A n A D C$ map $\left(A D C=1.65 \pm 0.18 \times 10^{-3} \mathrm{~mm}^{2} / \mathrm{second}\right)$. The circle marks the tumor's location. F: Magnetic resonance image showing the shrunken tumor 3 years after GKS.

will allow more precise measurements within smaller voxel volumes.

We examined moderate or large tumors in this study, because in cases of small tumors, the osseous component of the skull base is an obstacle for DW imaging. We suggest that DW imaging and ADC measurements be included in routine MR imaging examinations to provide additional information to differentiate tumor responses and to help evaluate equivocal MR imaging findings. This study does not show the proliferative potential of remaining tumor cells, which may be independent of water. Therefore, MR spectroscopy, which provides insight into tumor metabolites, may be applied to further enhance the evaluation of the tumor response to treatment.

Measurement of ADCs may be useful for evaluating treatment results before any definite volume change is noted on imaging studies and to distinguish a radiation effect from a tumor recurrence in cases in which imaging studies are not definitive, as in some patients with increased tumor volumes or no volume change. We suggest that ADC measurements be included in routine MR imaging examinations for the evaluation of GKS results.

\section{Disclosure}

The authors report no conflict of interest concerning the materials or methods used in this study or the findings specified in this paper.

Author contributions to the study and manuscript preparation include the following. Conception and design: Huang, CC Chuang. Acquisition of data: CC Chuang, Chang. Analysis and interpretation of data: CC Chuang. Critically revising the article: all authors. Reviewed submitted version of manuscript: all authors. Approved the final version of the manuscript on behalf of all authors: Huang. Statistical analysis: Huang, CC Chuang, Chang, Tu. Administrative/ technical/material support: Huang, CC Chuang, Chang, KS Chuang. Study supervision: Huang, KS Chuang.

\section{References}

1. Adler JR, Cox RS, Kaplan I, Martin DP: Stereotactic radiosurgical treatment of brain metastases. J Neurosurg 76:444449, 1992

2. Brown PD, Brown CA, Pollock BE, Gorman DA, Foote RL: Stereotactic radiosurgery for patients with "radioresistant" brain metastases. Neurosurgery 51:656-667, 2002

3. Chenevert TL, McKeever PE, Ross BD: Monitoring early response of experimental brain tumors to therapy using diffusion magnetic resonance imaging. Clin Cancer Res 3:14571466, 1997

4. Chenevert TL, Stegman LD, Taylor JM, Robertson PL, Greenberg HS, Rehemtulla A, et al: Diffusion magnetic resonance imaging: an early surrogate marker of therapeutic efficacy in brain tumors. J Natl Cancer Inst 92:2029-2036, 2000

5. Huang CF, Chou HH, Tu HT, Yang MS, Lee JK, Lin LY: Diffusion magnetic resonance imaging as an evaluation of the response of brain metastases treated by stereotactic radiosurgery. Surg Neurol 69:62-68, 2008

6. Iwai Y, Yamanaka K, Shiotani M, Uyama T: Radiosurgery for acoustic neuromas: results of low-dose treatment. Neurosurgery 53:282-288, 2003 
7. Kauppinen RA: Monitoring cytotoxic tumour treatment response by diffusion magnetic resonance imaging and proton spectroscopy. NMR Biomed 15:6-17, 2002

8. Kondziolka D, Lunsford LD, McLaughlin MR, Flickinger JC: Long-term outcomes after radiosurgery for acoustic neuromas. N Engl J Med 339:1426-1433, 1998

9. Krabbe K, Gideon P, Wagn P, Hansen U, Thomsen C, Madsen F: MR diffusion imaging of human intracranial tumours. Neuroradiology 39:483-489, 1997

10. Leksell L: Stereotactic radiosurgery. J Neurol Neurosurg Psychiatry 46:797-803, 1983

11. Linskey ME, Martinez AJ, Kondziolka D, Flickinger JC, Maitz $\mathrm{AH}$, Whiteside $\mathrm{T}$, et al: The radiobiology of human acoustic schwannoma xenografts after stereotactic radiosurgery evaluated in the subrenal capsule of athymic mice. J Neurosurg 78:645-653, 1993

12. Okamoto K, Ito J, Ishikawa K, Sakai K, Tokiguchi S: Diffusion-weighted echo-planar MR imaging in differential diagnosis of brain tumors and tumor-like conditions. Eur Radiol 10:1342-1350, 2000

13. Petit JH, Hudes RS, Chen TT, Eisenberg HM, Simard JM, Chin LS: Reduced-dose radiosurgery for vestibular schwannomas. Neurosurgery 49:1299-1307, 2001

14. Sener RN: Diffusion magnetic resonance imaging of solid vestibular schwannomas. J Comput Assist Tomogr 27:249_ 252,2003
15. Sener RN: Diffusion MRI: apparent diffusion coefficient (ADC) values in the normal brain and a classification of brain disorders based on ADC values. Comput Med Imaging Graph 25:299-326, 2001

16. Sugahara T, Korogi Y, Kochi M, Ikushima I, Shigematu Y, Hirai T, et al: Usefulness of diffusion-weighted MRI with echoplanar technique in the evaluation of cellularity in gliomas. $\mathbf{J}$ Magn Reson Imaging 9:53-60, 1999

17. Yamasaki F, Kurisu K, Satoh K, Arita K, Sugiyama K, Ohtaki $\mathrm{M}$, et al: Apparent diffusion coefficient of human brain tumors at MR imaging. Radiology 235:985-991, 2005

Manuscript submitted May 15, 2012.

Accepted July 10, 2012.

Portions of this work were presented in abstract form at the 16th International Meeting of the Leksell Gamma Knife Society, Sydney, Australia, March 25-29, 2012.

Please include this information when citing this paper: DOI: 10.3171/2012.7.GKS121003.

Address correspondence to: Chuan-Fu Huang, M.D., Department of Neurosurgery, China Medical University Hospital, 2 YuhDer Road, Taichung City, Taiwan 40447, R.O.C. email: gkf0218@ yahoo.com.tw. 